\title{
Nonlinear gas oscillations in pipes. Part 1. Theory
}

\author{
By J. JIMENEZ \\ Applied Mathematics, California Institute of Technology
}

(Received 11 September 1972 and in revised form 5 February 1973)

The problem of forced acoustic oscillations in a pipe is studied theoretically. The oscillations are produced by a moving piston in one end of the pipe, while a variety of boundary conditions ranging from a completely closed to a completely open mouth at the other end are considered. All these boundary conditions are modelled by two parameters: a length correction and a reflexion coefficient equivalent to the acoustic impedance.

The linear theory predicts large amplitudes near resonance and nonlinear effects become crucially important. By expanding the equations of motion in a series in the Mach number, both the amplitude and wave form of the oscillation are predicted there.

In both the open- and closed-end cases the need for shock waves in some range of parameters is found. The amplitude of the oscillation is different for the two cases, however, being proportional to the square root of the piston amplitude in the closed-end case and to the cube root for the open end.

\section{Introduction}

The problem we consider is the oscillation of a gas inside a pipe whose length is $L$, and whose transverse dimension is small with respect to the length. At one end of the pipe $(x=0)$ a piston executes small harmonic oscillations with a frequency that we choose to be of the order of the resonant frequency associated with $L$. At the other end ( $x=L$ ) we want to model a range of physical conditions progressing from a completely closed to a completely open tube, including different kinds of perforated end plates or other mouth configurations, thereby producing varying amounts of coupling to the room.

If the pipe we study is slender a reasonable assumption is that there exists an equivalent one-dimensional problem, approximating the actual one and characterized by 'effective' cross-sectional conditions. This is usually a good assumption everywhere in the pipe except near the mouth section, where the matching of the flow in the tube to the three-dimensional flow in the room gives rise to local transverse effects. The classical way to model these effects in linear acoustics is the use of an equivalent impedance of the end section (Morse \& Ingard 1968, p. 467). The real and imaginary parts of this impedance can be considered quite separately.

The imaginary part corresponds simply to a length correction. The effective length of the pipe is different from the real length, and this difference accounts for part of the two-dimensional effects at the mouth section. There are some 
classical theoretical results (Rayleigh $1945, \S 307$ ) aiming to predict the value of this correction, based on assumptions like potential flow near the pipe end and no viscous effects. In the real world, however, these assumptions are hardly every fulfilled except for the smallest amplitudes of the gas oscillations. In any case, as the correction arises from transverse effects, the general order of magnitude can be expected to be no more than a few pipe diameters, and this should cause no qualitative difference in the behaviour for a slender pipe.

In a similar way, the real part of the impedance can be interpreted as a partial reflexion coefficient. The general idea is that, from some diameters away, the pipe end should look like a virtual plane section. A long wave running into this section from the pipe is partially reflected and partially transmitted, or somehow dissipated. The simplest model for this process, which was also proposed by Seymour \& Mortell (1973), is to assume that the reflected wave is proportional to the incoming one, with a proportionality coefficient ranging between +1 and -1 . In the same fashion as with the length correction there are theoretical estimates for the value of this factor, but they cannot be trusted for waves of any reasonable amplitude.

In summary, although the impedance model was created for linear oscillations, it still provides a very compact way of treating a wide range of physical cases. In fact, there have been several attempts to justify its use in the treatment of acoustically absorbent materials at high radiation intensities where nonlinear effects appear. Thus, in 1967, Ingard \& Ising, using experiments with resonators, proved that the behaviour of orifices could be successfully approximated at moderate intensities by the use of an amplitude-dependent impedance coefficient.

The experiments undertaken to complement the present work, to be reported in part 2 of this series, have been carried out at much higher intensities than in previous cases. Therefore it is of interest to check them against the predictions of the impedance model and, in that way, to judge the validity of the model itself.

In this paper, we therefore develop a nonlinear theory applicable to those cases in which resonance peaks occur with sufficient amplitude that linear analysis is inadequate. The first suggestion of the importance of nonlinear effects in these cases was made by Lettau (1939), who observed the appearance of travelling shock waves near the linear resonant frequency in both closed and open tubes.

Using these experiments as a guide Betchov (1958) constructed a theoretical solution of the flow in a closed pipe, in which, with a few well-chosen assumptions on the general form of the solution, he was able to prove that the nonlinearity of the equations alone bounds the resonant amplitude away from infinity without recourse to dissipation, as well as to compute wave forms in qualitative agreement with the experimental results.

Saenger \& Hudson (1960) further refined the experimental observations and attempted to account theoretically for the effects of viscous shear and heat conduction. Finally, Chester (1964) developed a consistent theory for the closed pipe in which, without any special assumption, the appearance and strength of the shock waves, as well as the detailed wave forms for all frequencies, were predicted. One important result of his paper was to show that the amplitude of 
the pressure oscillation is of $O\left(\delta^{\frac{1}{2}}\right)$, whereas the piston amplitude is much smaller, of $O(\delta)$. Temkin (1968) obtained still more experimental data on the closed tube and gave a simple but elegant account of the different effects present in the problem using energy balance considerations.

Wijngaarden (1968) treated the case of an open pipe at resonance using a nonlinear boundary condition in which viscous dissipation caused by flow separation at the pipe exit was assumed to dominate radiation losses and, in fact, the nonlinear behaviour of the gas in the pipe itself. This is probably true for some range of the geometrical parameters of the pipe and represents the opposite extreme to the case treated in the present work. The pressure amplitude he derived is again of $O\left(\delta^{\frac{1}{2}}\right)$ but this is governed primarily by the dissipation boundary condition.

Mortell (1971) attempted a straightforward generalization of Chester's analysis of the closed pipe to other cases of nonlinear oscillations, including the open pipe with a perfectly reflecting exit. It turns out, however, that the method does not generalize to this case without special precautions, and Mortell derived the wrong result. In particular, the amplitude of the oscillation was mistakenly given as of $O\left(\delta^{\frac{1}{2}}\right)$.

The first author to point to the correct result was Collins (1971), who studied the problem of a nonlinear wave equation applied to the vibration of a string. He correctly expanded the equation in terms of the resulting amplitude, instead of the forcing amplitude, pointed out the similarity to the open pipe, and predicted the resulting amplitude to be of $O\left(\delta^{\frac{1}{3}}\right)$. He did not present detailed calculations for the gasdynamic case and, in particular, failed to point out the presence of shock waves in the solution.

A closely related class of problems was treated by Chu \& Ying (1963), when they studied thermally induced oscillations in closed pipes. They used a characteristies perturbation procedure due to Lin (1954) which is very close to the one used in this paper.

Finally Seymour \& Mortell (1973) describe an extension of Chester's method to a nearly closed pipe with radiation damping and obtain results similar to the ones in $\S 4$ of this paper.

\section{General equations}

Consider a pipe along the $x$ axis. The passive end of the pipe is located at $x=L$. At $x=0$ a piston oscillates according to the law

$$
x=-l \cos \varpi t
$$

and this causes the gas to vibrate around an equilibrium state given by a sound speed $a_{0}$ and zero gas velocity. By making velocity and time non-dimensional with $a_{0}$ and $\pi / \varpi$ respectively, we have the sound speed fluctuating about the value 1 and the period of the oscillation fixed for all driving frequencies and equal to 2.

When we change the driving frequency, however, the unit of length changes and the pipe has a variable length in the new co-ordinates, with the passive end located at

$$
x=\varpi L / \pi a_{0} \equiv \omega / \pi,
$$



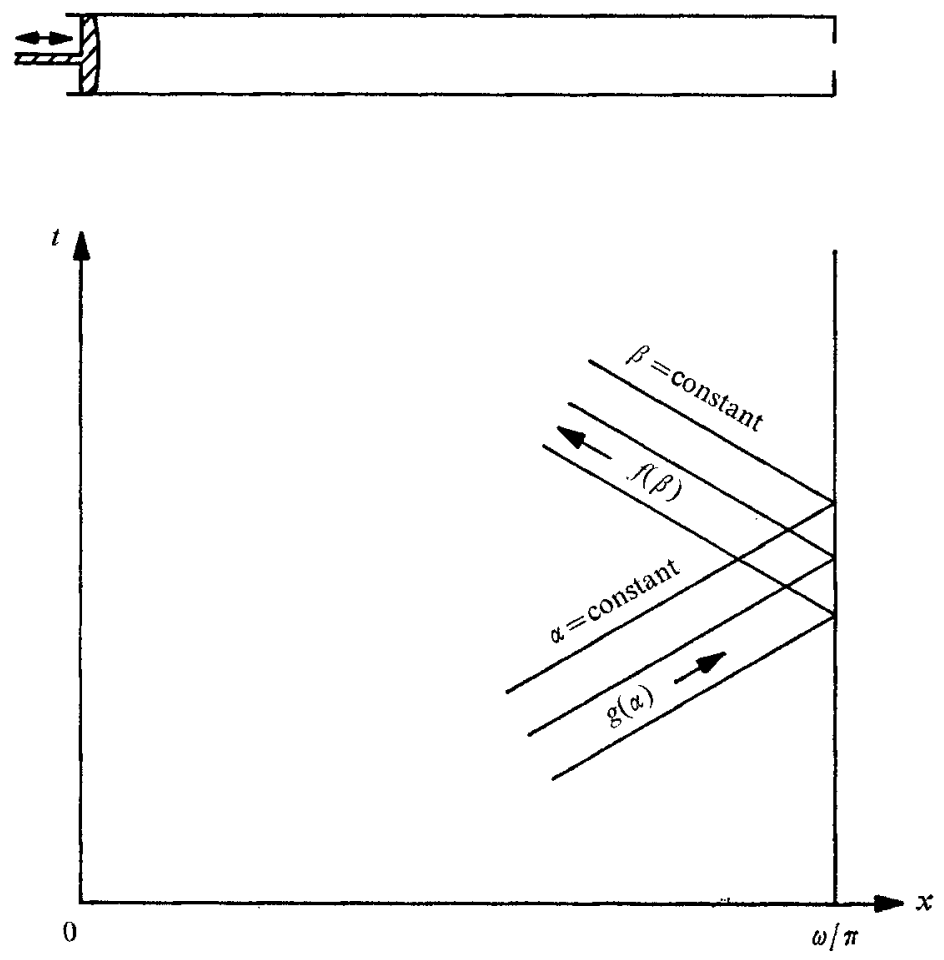

FIGURE 1. Pipe configuration and non-dimensional co-ordinates.

where

$$
\omega \equiv \varpi L / a_{0}
$$

is a non-dimensional measure of the forcing frequency. The motion of the piston, however, has constant frequency, and is given by

$$
x=-(\delta / \pi) \cos \pi t
$$

The parameter $\delta$, defined by

$$
\delta=\varpi l / a_{0} \equiv \omega l / L
$$

is now a good indicator of the strength of the forcing terms, and is in fact a Mach number for the motion of the piston and for the motion of the gas near the piston.

One might assume then that $\delta$ also measures the strength of the gas oscillation everywhere on the pipe, so that if $\delta$ is small the linearized acoustic equations would be applicable. If one tries to do that, one gets consistent results for all values of $\omega$ except for those pipe lengths which are near resonance with the piston frequency. At these values of $\omega$ the motion of the gas is much larger, in general, than $\delta$ and it becomes necessary to include higher order nonlinear terms in the equations of motion. It should be noted, therefore, that the correct expansion parameter should be the typical Mach number of the gas, which we take to be $\epsilon$, not the velocity parameter of the piston $\delta$, and that one of the aims of the theory should be to find the relation between $\delta$ and $\epsilon$.

We first write the general equations of motion, considering the gas to be ideal and isentropic, and the motion to be one-dimensional. The velocity of the gas is $u$ 
and the sound speed $1+a ; x$ and $t$ are Eulerian co-ordinates and $\alpha$ and $\beta$ the corresponding characteristic co-ordinates. Under these circumstances the equations and boundary conditions are (Courant \& Friedrichs 1948, p. 80)

$$
\begin{gathered}
\partial x / \partial \alpha=(u-a-1) \partial t / \partial \alpha \\
\partial x / \partial \beta=(u+a+1) \partial t / \partial \beta, \\
{[2 /(\gamma-1)] a-u=2 f(\beta), \quad[2 /(\gamma-1)] a+u=2 g(\alpha) ;} \\
x=0, \quad t=\alpha \quad \text { on } \quad \alpha=\beta ; \\
u=g(\alpha)-f(\beta)=\delta \sin \pi t \quad \text { on } \quad x=-(\delta / \pi) \cos \pi t .
\end{gathered}
$$

Equations (2.5) define the geometrical co-ordinates $x$ and $t$ in the characteristic plane, and (2.7) makes the definition unique by choosing $\alpha$ and $\beta$ to be the time $t$ at $x=0$. In the pipe $\alpha$ is constant along the $C^{+}$characteristics and $\beta$ along the $C^{-}$ characteristics. Equations (2.6) define the invariants riding each family of characteristics. In particular $g(\alpha)$ can be considered as a "simple" wave going to the right, and $f(\beta)$ as the left-going reflected wave. Equation (2.8) is the boundary condition at the piston.

The only remaining equation is the boundary condition at the passive end of the pipe and, following the discussion in the introduction, we assume it to be

$$
f(\beta)=b g(\alpha) \text { on } x=\omega / \pi,
$$

where $b$ is a number between +1 and -1 which gives the fraction of the rightgoing wave that is reflected back into the tube. The condition that $b$ be bounded between +1 and -1 obviously means that no energy is created at the passive end, or, more specifically, that any radiated energy is transmitted from the pipe to the room, and not vice versa. It is easily seen that the value $b=1$ corresponds to zero velocity at the end section and is equivalent to a perfectly closed pipe, while $b=-1$ implies $a=0$ and represents an ideally open end, at which the pressure is always equal to room pressure.

Equations (2.5)-(2.9), plus the periodicity condition which says that we are looking for a steady oscillation of the same period as the piston, completely define the problem.

If we assume now that $u$ and $a$ are of $O(\delta)$ and $\delta \ll 1$, we can neglect the secondorder terms in (2.5) and apply the piston condition at $x=0$. The result is the classical linear theory. The characteristics are parallel straight lines and the solution for $g(\alpha)$ is sinusoidal with amplitude

$$
g_{\max }=\delta\left[1+b^{2}-2 b \cos 2 \omega\right]^{-\frac{1}{2}}
$$

When $b$ is close to \pm 1 this amplitude has sharp resonance peaks in $\omega$. There the oscillation is much larger than $O(\delta)$, and nonlinear effects may be expected to be important. For $b$ far from these values, however, the linear theory is correct for $\delta$ small, as only broad resonance peaks occur and amplitudes remain of order $\delta$.

A particularly interesting case arises for $b=0$, when the amplitude of the 
oscillation is completely independent of frequency. This is, of course, because no wave is reflected from the pipe end and the oscillation is just the simple wave produced by the piston, travelling undisturbed to infinity. An experimental approximation to this case should be a plate with a $50 \%$ hole in it.

Therefore, the only regions in the $b, \omega$ plane where linear theory should not be expected to hold are very small regions whose extent logically depends on the size of $\epsilon$. Their location can be derived from the linear theory and falls into two families. Almost-closed pipes; where $b$ is near +1 , have resonance peaks at $\omega \simeq \pi$, $2 \pi, 3 \pi, \ldots$, and almost-open pipes, with $b$ near -1 , are resonant at $\omega \simeq \frac{1}{2} \pi, \frac{3}{2} \pi$, $\frac{5}{2} \pi, \ldots$

An asymptotic theory trying to explore these regions for small $\epsilon$ should include, then, expansions for $b$ and $\omega$ as well as for the other quantities. We develop such a theory in the following sections, dealing primarily with the first resonance peaks for both the open and closed cases.

\section{Perturbation scheme}

\subsection{Basic expansions}

The first question to be solved is the relation between $\epsilon$, the Mach number in the gas, and $\delta$, the piston motion. We have seen that assuming both to be of the same order leads to a first-order solution for the velocity that is inadequate near resonance. This suggests that the nonlinear behaviour of the wave should be made to balance the forcing term, and as this nonlinearity can be expressed as a power series in $\epsilon$, it is logical to expect that $\delta$ can be equated to some integer power of $\epsilon$ :

$$
\delta=\epsilon^{N} \text {. }
$$

The value of $N$ has to be assumed at the beginning of the perturbation procedure, and the test of the assumption is consistency in the resulting analysis. If the assumed value of $N$ is too low, we will get essentially the linear theory $(N=1)$, and if too large the solution will be identically zero to first order, contrary to the assumption that $\epsilon$ is the order of the oscillation.

It seems to be important to work the problem directly in characteristic coordinates, as working with approximate characteristics in the $x, t$ plane gives rise to secular terms in the solution. The problem seems to be the same as that with weakly nonlinear oscillators, where the period depends on the amplitude, and the correct perturbation procedure is by Poincaré's method. The use of characteristic co-ordinates here corresponds to the expansion of the independent variables used in that case.

If $\alpha$ and $\beta$ are, then, considered as the independent variables, the appropriate expansions for $u$ and $\alpha$ are

$$
\left.\begin{array}{l}
a /(\gamma-1)-\frac{1}{2} u=f(\beta)=\epsilon f_{1}(\beta)+\epsilon^{2} f_{2}(\beta)+\ldots, \\
a /(\gamma-1)+\frac{1}{2} u=g(\alpha)=\epsilon g_{1}(\alpha)+\epsilon^{2} g_{2}(\alpha)+\ldots .
\end{array}\right\}
$$

The geometrical co-ordinates $x$ and $t$ must too be expanded in powers of $\epsilon$. We take

$$
\left.\begin{array}{rl}
x & =x_{0}(\alpha, \beta)+\epsilon x_{1}+\epsilon^{2} x_{2}+\ldots, \\
t & =t_{0}(\alpha, \beta)+\epsilon t_{1}+\epsilon^{2} t_{2}+\ldots
\end{array}\right\}
$$


Using these expansions in the equations for the characteristics (2.5) and separating orders, we get for all $n$

$$
\left.\begin{array}{l}
\frac{\partial}{\partial \alpha}\left(x_{n}+t_{n}\right)=-\sum_{i=1}^{n}\left\{\frac{\gamma+1}{2} f_{i}(\beta)+\frac{\gamma-3}{2} g_{i}(\alpha)\right\} \frac{\partial}{\partial \alpha} t_{n-i}, \\
\frac{\partial}{\partial \beta}\left(x_{n}-t_{n}\right)=\sum_{i=1}^{n}\left\{\frac{\gamma-3}{2} f_{i}(\beta)+\frac{\gamma+1}{2} g_{i}(\alpha)\right\} \frac{\partial}{\partial \beta} t_{n-i^{*}}
\end{array}\right\}
$$

The corresponding boundary conditions (2.7) are expanded to:

$$
\left.\begin{array}{c}
x_{0}=0, t_{0}=\alpha, \\
x_{n}=t_{n}=0, \quad n \geqslant 1,
\end{array}\right\} \text { at } \alpha=\beta .
$$

The system (3.4) with (3.5) can be solved recursively in terms of the f's and $g$ 's. To the first two orders $x$ and $t$ are given by

$$
x_{0}=\frac{1}{2}(\beta-\alpha), \quad t_{0}=\frac{1}{2}(\beta+\alpha),
$$

which correspond to the linear characteristics, and

$$
\begin{aligned}
& \left.\begin{array}{l}
x_{1}=\frac{1}{8}(\gamma+1)(\beta-\alpha)\left[f_{1}(\beta)+g_{1}(\alpha)\right]+\frac{1}{8}(\gamma-3)\left[\psi_{1}(\beta)+\phi_{1}(\beta)-\psi_{1}(\alpha)-\phi_{1}(\alpha)\right], \\
t_{1}=\frac{1}{8}(\gamma+1)(\beta-\alpha)\left[f_{1}(\beta)-g_{1}(\alpha)\right]+\frac{1}{8}(\gamma-3)\left[\psi_{1}(\beta)-\phi_{1}(\beta)-\psi_{1}(\alpha)+\phi_{1}(\alpha)\right],
\end{array}\right\} \\
& \text { where } \quad \psi_{i}(\alpha)=\int^{\alpha} g_{i}(\xi) d \xi, \quad \phi_{i}(\alpha)=\int^{\alpha} f_{i}(\xi) d \xi .
\end{aligned}
$$

Similarly, $x_{2}$ and $t_{2}$ can be computed as functions of $f_{1}, g_{1}, f_{2}$ and $g_{2}$, and $x_{n}$ and $t_{n}$ as functions of the $f_{i}$ and $g_{i}$ up to $i=n$ only. This fact makes for an ordered expansion procedure in which higher order terms do not 'feed back' to lower orders.

It should be noted that the system (3.4) with (3.5) together with the definitions of $f_{i}$ and $g_{i}$ in (3.2) contain all the equations of motion of the gas and, in particular, all the nonlinearities of the problem. All that remains now is to apply the boundary conditions to find $f$ and $g$.

The first boundary condition is the one at the piston (2.8). With the assumption made above on $\delta$, we can write $(2.8)$ to $O\left(\epsilon^{N}\right)$ as

$$
\left.\begin{array}{rl}
g_{i}(\alpha) & =f_{i}(\alpha), \quad i<N \\
g_{N}(\alpha) & =f_{N}(\alpha)+\sin \pi \alpha .
\end{array}\right\}
$$

This condition halves the number of unknown functions by eliminating the $g_{i}$. Physically, the wave in the pipe does not 'see' the piston up to order $N$, and if $N>1$, the solution is essentially a free standing wave, with its shape determined by the piston only through the higher order terms. As $N \doteq 1$ corresponds to the classical linear theory, near resonance we must actually have the situation $N>1$.

Next it is necessary to introduce the condition (2.9) at the passive end; this condition includes the parameters $\omega$ and $b$. From the discussion in the last section we know that these parameters should be expanded around their values at resonance:

$$
\left.\begin{array}{c}
\omega=\omega_{0}\left[1+\epsilon \omega_{1}+\epsilon^{2} \omega_{2}+\ldots\right], \\
b=b_{0}\left[1-\epsilon b_{1}-\epsilon^{2} b_{2}+\ldots\right],
\end{array}\right\}
$$


where the two first resonance peaks correspond to

$$
\left.\begin{array}{l}
b_{0}=-1, \quad \omega_{0}=\frac{1}{2} \pi \text { for an open end, } \\
b_{0}=+1, \quad \omega_{0}=\pi \text { for a closed end. }
\end{array}\right\}
$$

The position $x=\omega / \pi$ of the passive end will now be mapped into a certain line in the $\alpha, \beta$ plane

$$
\beta=\tilde{\beta}(\alpha)=\beta_{0}(\alpha)+\epsilon \beta_{1}(\alpha)+\epsilon^{2} \beta_{2}(\alpha)+\ldots
$$

To compute it first expand

$$
x=\omega / \pi=\left(\omega_{0} / \pi\right)\left(1+\epsilon \omega_{1}+\epsilon^{2} \omega_{2}\right)=x_{0}(\tilde{\beta}, \alpha)+\epsilon x_{1}(\tilde{\beta}, \alpha)+\ldots
$$

Then substitute (3.12) in the right-hand side of (3.13), expand the $x_{i}(\tilde{\beta}, \alpha)$ in Taylor series and equate like orders to solve for $\tilde{\beta}$ :

$$
\left.\begin{array}{l}
\beta_{0}(\alpha)=\alpha+\left(2 \omega_{0} / \pi\right), \quad \beta_{1}(\alpha)=\left(2 \omega_{0} / \pi\right) \omega_{1}-2 x_{1}\left(\alpha, \beta_{0},\right) \\
\beta_{2}(\alpha)=\left(2 \omega_{0} / \pi\right) \omega_{2}-2 x_{2}\left(\alpha, \beta_{0}\right)-2 \beta_{1}(\alpha)(\partial / \partial \beta) x_{1}\left(\alpha, \beta_{0}\right) .
\end{array}\right\}
$$

Equations (3.14) can be expressed in terms of the $f_{i}$. Once again, the solution is ordered in the sense that $\beta_{n}(\alpha)$ does not contain terms from orders higher than $n$. Finally, we use all these expansions in the reflexion condition (2.9), which can now be written as

$$
b\left[f(\alpha)+\epsilon^{N} \sin \pi \alpha\right]=f(\tilde{\beta}) .
$$

Expanding the right-hand side in Taylor series and separating orders yields

$$
\begin{gathered}
f_{1}(\alpha)-b_{0} f_{1}\left(\beta_{0}\right)=0 \quad \text { to } O(\epsilon) \\
f_{2}(\alpha)-b_{0} f_{2}\left(\beta_{0}\right)=b_{1} f_{1}(\alpha)+b_{0} \beta_{1}(\alpha) f_{1}^{\prime}\left(\beta_{0}\right)-\delta_{N 2} \sin \pi \alpha \text { to } O\left(\epsilon^{2}\right) ; \\
f_{3}(\alpha)-b_{0} f_{3}\left(\beta_{0}\right)=\text { funct }\left\{f_{1}, f_{2}\right\}-\delta_{N 3} \sin \pi \alpha \text { to } O\left(\epsilon^{3}\right)
\end{gathered}
$$

where $\delta_{N M}$ denotes the Kronecker delta.

This hierarchy by itself does not allow the calculation of the $f_{i}$, unless we impose some conditions of the solution, which, in fact, correspond to the initial conditions necessary in the hyperbolic problem. As we are looking for steady oscillations, we impose the condition that the solution must be periodic with the same period as the piston. But, since $\alpha$ and $\beta$ correspond to real time at $x=0$, periodicity in time means directly periodicity in $\alpha$ and $\beta$. So, the desired condition is that the $f_{i}(\alpha)$ be periodic with period 2 , i.e.

$$
f_{i}(\alpha)=f_{i}(\alpha+2) \text { for all } i
$$

These conditions enable us to eliminate the left-hand sides from (3.15) and get finally a set of equations for $f$. The details of the elimination vary slightly from the open- to the closed-end case, so the two cases must be considered separately.

\subsection{Closed-end case}

Doing first the closed-end case, we start by assuming that $N=2$ ( $N=1$ would give the classical linear theory). From (3.11) and (3.14), $\beta_{0}=\alpha+2$, and the lefthand sides of (3.15) are of the type

$$
f_{i}(\alpha)-f_{i}(\alpha+2)
$$


and, because of periodicity, are all identically zero. The desired set of equations is then given by the right-hand sides of (3.15) equated to zero.

The equation for $O(\epsilon)$, equation (3.15a), is satisfied identically and gives no information, but the second equation, of $O\left(\epsilon^{2}\right)$, gives an equation for $f_{1}$ :

where

$$
\left.\begin{array}{c}
2\left[\hat{\omega}_{1}-\frac{1}{2}(\gamma+1) f_{1}\right] f_{1}^{\prime}+b_{1} f_{1}=\sin \pi \alpha, \\
\hat{\omega}_{1}=\omega_{1}-\frac{1}{2}(\gamma-3)\left\langle f_{1}\right\rangle,
\end{array}\right\}
$$

with

$$
\left\langle f_{1}\right\rangle \equiv \frac{1}{2} \int_{0}^{2} f_{1}(\xi) d \xi
$$

representing the mean value of $f_{1}$ over one period. For $b_{1}=0$, the completely closed end, (3.17) reduces to the equation obtained by Chester (1964). We delay the analysis of (3.17) until the next section.

\subsection{Open-end case}

For an open end $b_{0}=-1$ and $\beta_{0}=\alpha+1$, so that the left-hand sides of (3.15) are of the type

$$
f_{i}(\alpha)+f_{i}(\alpha+1)
$$

and do not vanish in general. However, if the $f_{i}$ have period 2 , the expressions in (3.19) have period 1, and that imposes restrictions on the right-hand sides, which give the desired equations.

In particular,

$$
[\text { R.H.S. }]_{i}(\alpha)=[\text { R.H.S. }]_{i}(\alpha+1)
$$

is an equation involving only the $f$ 's up to order $i-1$. From the first order in (3.15) we get

$$
f_{1}(\alpha+1)=-f_{1}(\alpha)
$$

so that the wave form changes sign as we advance a semi-period. This is important because it means that any shock wave in the solution implies an expansion shock half a period away. It should be remembered that the original equations were isentropic, so expansion discontinuities are not really inconsistent in lower order approximations. On the other hand, we shall see later that $f_{1}$ cannot be calculated until we include third-order effects, so that the isentropic assumption breaks down with the presence of shocks of $O(\epsilon)$. We shall come back later to this problem.

Assume now that $N=2$, and consider $O\left(\epsilon^{2}\right)$ terms in (3.15). Forming the equation corresponding to (3.20) we get, after some algebra,

$$
b_{1} f_{1}+\omega_{1} f_{1}^{\prime}=\sin \pi \alpha,
$$

which is linear and has no bounded solution for the case $b_{1}=\omega_{1}=0$, corresponding to resonance. That means that the assumption $N=2$ was wrong and that we should go to higher order. For $N>2$, equation (3.22) appears with right-hand side zero. Therefore, $b_{1}$ and $\omega_{1}$ have to vanish in the resonance band, as the homogeneous part of (3.22) has no non-trivial periodic solution.

The physical interpretation of this result is that the resonance band in this case is, at most, of $O\left(\epsilon^{2}\right)$ and the piston motion of $O\left(\epsilon^{3}\right)$. Outside this region we recover again the linear theory. 
Assuming then that

$$
N=3, \quad b_{1}=\omega_{1}=0,
$$

and repeating the process for (3.15) up to third order, we get, after a great deal of algebra, an equation for $f_{1}$ :

where

$$
\left.\begin{array}{c}
{\left[\hat{\omega}_{2}+\frac{1}{16}(\gamma+1)(3 \gamma+7) f_{1}^{2}\right] f_{1}^{\prime}+b_{2} f_{1}=\sin \pi \alpha,} \\
\hat{\omega}_{2}=\omega_{2}+\frac{1}{16}(3-\gamma)(13-3 \gamma)\left\langle f_{1}^{2}\right\rangle,
\end{array}\right\}
$$

with

$$
\left\langle f_{1}^{2}\right\rangle=\frac{1}{2} \int_{0}^{2} f_{1}^{2} d \xi=\int_{0}^{1} f_{1}^{2} d \xi .
$$

It is, in fact, easy to prove from (3.15) that $\left\langle f_{1}^{2}\right\rangle$ is proportional to $\left\langle f_{2}\right\rangle$, and so to $\langle f\rangle$, as the mean value of $f_{1}$ over one period is zero because of (3.21).

The correction to the frequency given by (3.24) in the open-end case is then of the same type as the one given in (3.17) for the closed end, and both can be interpreted as a shift in the linear resonant frequency due to the difference between the real mean pressure and the pressure defined $a$ priori as mean.

It is interesting, in fact, to examine the validity of the separation of the pressure (sound speed) wave form into a mean value and a perturbation, particularly as this separation is usually not clear-cut in nonlinear problems. In this case, however, a clear definition of $a=0$ is introduced by the boundary condition of $x=L$, as this condition is linear. In fact the reflexion condition is equivalent to making $a \sim u$, except for $b$ exactly equal to 1 . With that exception, then, $a=0$ corresponds to the state at the pipe exit when $u=0$, and that can, in principle, be related to ambient conditions.

The special case $b=1$ corresponds to the completely closed pipe and the gas in the tube, having no connexion with the atmosphere, does not have any clearly defined mean pressure. This is reflected in (3.17), where $\left\langle f_{1}\right\rangle$ can be determined for all cases except $b_{1}=0$. Integrating (3.17) over one period, we get

$$
2 \widehat{\omega}_{1}\left\langle f_{1}^{\prime}\right\rangle-(\gamma+1)\left\langle f_{1} f_{1}^{\prime}\right\rangle+b_{1}\left\langle f_{1}\right\rangle=\langle\sin \pi \alpha\rangle \text {. }
$$

The first two terms are perfect differentials and vanish because of periodicity as does the right-hand side, so we get

$$
b_{1}\left\langle f_{1}\right\rangle=0 .
$$

If $b_{1} \neq 0, f_{1}$ has to have zero mean. If $b_{1}=0,\left\langle f_{1}\right\rangle$ is not fixed and we know from the previous discussion that we can define it arbitrarily. To preserve continuity of the solution with $b_{1}$ we define it as

$$
\left\langle f_{1}\right\rangle=0
$$

for all $b_{1}$, and use this to simplify (3.17).

\section{Analysis of the closed-end case}

We now come to the problem of actually solving (3.17) and (3.24) to find the response of the system near the two resonances. The first difficulty is that both equations are singular for some values of the parameters, and the effect of 
these singularities must be studied before we attempt a numerical treatment of the equations.

Consider first (3.17), representing the closed-end case. Following the discussion at the end of last section, it can be simplified to

$$
\left[2 \omega_{1}-(\gamma+1) f_{1}\right] f_{1}^{\prime}+b_{1} f_{1}=\sin \pi \alpha
$$

with the boundary conditions

$$
\begin{gathered}
f_{1}(\alpha+2)=f_{1}(\alpha), \\
\left\langle f_{1}\right\rangle \equiv \frac{1}{2} \int_{0}^{2} f_{1}(\xi) d \xi=0 .
\end{gathered}
$$

For all $b_{1} \neq 0$, condition (4.3) is redundant and follows directly from periodicity. In numerical calculations, moreover, (4.2) proves to be much easier to use, as it reduces the order of the problem by one. When $b_{1}=0$, however, (4.2) is automatically satisfied and (4.3) must be used. In this case, though, the equation can be integrated exactly, and there is no need for numerical work.

It may be as well at this point to remember the significance of the parameters $b_{1}$ and $\omega_{1}$. To do that we rewrite the expansions for $\omega$ and $b$ as

$$
\omega \simeq \pi\left(1+\epsilon \omega_{1}\right), \quad b \simeq 1-\epsilon b_{1},
$$

and note that $\omega_{1}$ represents the distance in frequency from exact resonance, and $b_{1}$ indicates the deviation of the end condition from the perfectly reflecting closed end or, in other words, the amount of wave radiated to the exterior. It follows that negative values of $b_{1}$ have no physical significance, and that the range of parameter to be studied is the upper half-plane in $b_{1}, \omega_{1}$ space.

The origin in this plane represents the perfectly closed pipe at resonance, and so, as we move away from it, we should approach the results of the linear theory. In fact, if in (4.1) we let $b_{1}$ or $\omega_{1}$ grow large, the nonlinear term can be neglected and we get asymptotically the linear result

$$
\left.\begin{array}{c}
f_{1}(\alpha)=-\left(4 \pi^{2} \omega_{1}^{2}+b_{1}^{2}\right)^{-\frac{1}{2}} \cos (\pi \alpha+\chi)+O\left(4 \pi^{2} \omega_{1}^{2}+b_{1}^{2}\right)^{-1}, \\
\chi=\tan ^{-1}\left(b_{1} / 2 \pi \omega_{1}\right)
\end{array}\right\}
$$

Another useful property of the system (4.1)-(4.3) is that it is invariant to the transformation

$$
f_{1}(\alpha) \rightarrow-f_{1}(-\alpha), \quad \omega_{1} \rightarrow-\omega_{1}, \quad b_{1} \rightarrow b_{1},
$$

and so it is possible to study the solution for $\omega_{1} \geqslant 0$ and extend it to all frequencies by using (4.6). In what follows we always assume that $\omega_{1}$ is positive.

We now solve (4.1) for the special case $b_{1}=0$, and, as stated before, the solution should reduce to the results given by Chester for the completely closed end. The equation can be integrated directly to

or

$$
\begin{gathered}
2 \omega_{1} f_{1}-\frac{1}{2}(\gamma+1) f_{1}^{2}+\pi^{-1} \cos \pi \alpha=\text { constant } \\
f_{1}=\frac{2 \omega_{1}}{\gamma+1}-\left[\frac{2}{\pi(\gamma+1)}\right]^{\frac{1}{2}}(K+\cos \pi \alpha)^{\frac{1}{2}}
\end{gathered}
$$




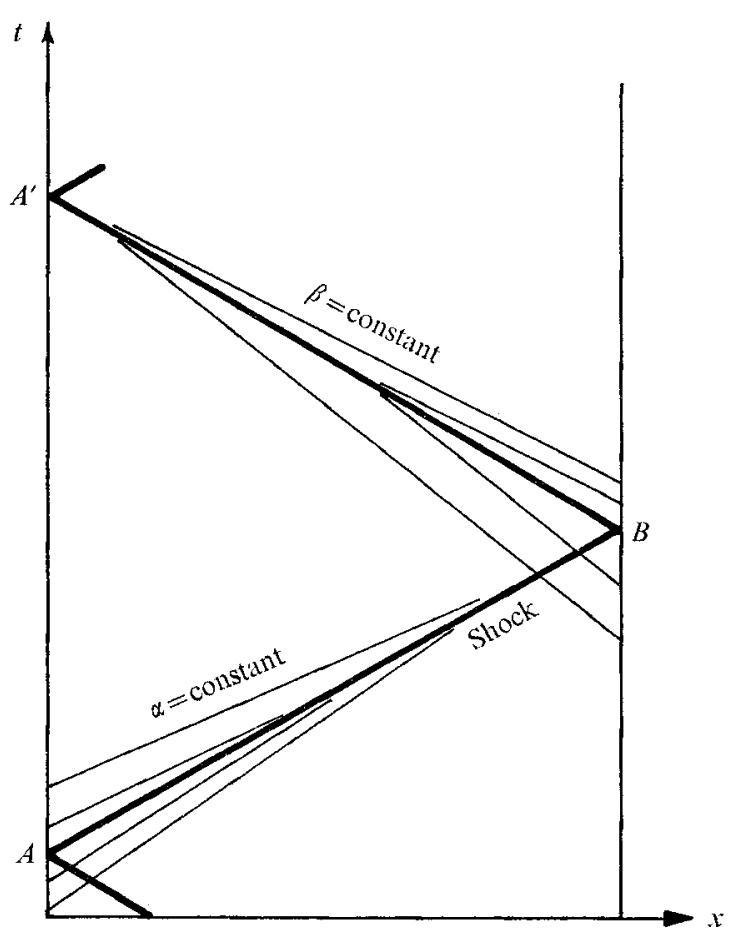

Figure 2. Shock geometry in the $x, t$ plane.

where $K$ is an integration constant to be determined with the help of (4.3). In attempting to do this, however, we get a transcendental equation in $K$ involving elliptic functions which has a real root only if

$$
\left|\omega_{1}\right| \geqslant \frac{2}{\pi}\left(\frac{\gamma+1}{\pi}\right)^{\frac{1}{2}}
$$

For all other values of $\omega_{1}$, then, there is no continuous solution satisfying (4.1) and (4.5). Chester (1964) interprets this fact as an indication of the appearance of shock waves in the flow, and this is confirmed by experiment.

In fact, in deriving (4.1), we only used the equations of motion up to $O\left(\epsilon^{2}\right)$, and to this order, a shock wave of amplitude of $O(\epsilon)$ produces no change in entropy and can be treated simply as a discontinuity in the solution (Courant \& Friedrichs $1948, p .156)$. The speed of propagation of this discontinuity is just the arithmetic mean of the wave velocities immediately in front of and behind it. This property, and the fact that a shock travelling along one set of characteristics does not modify waves travelling along the other set, contains the complete shock relations to the order needed. By using periodicity, the jump conditions across the shock discontinuity can now be determined.

Denote by superscripts + and - the conditions in front of and behind the discontinuity and consider first a shock, such as $A B$, travelling to the right (see figure 2). To first order, this shock is always located at a given value of $\alpha$, say $\alpha_{s}$, and its speed is

$$
V_{s}=1+\epsilon V_{1}=1+\frac{1}{2} \epsilon\left[\left(u_{1}+a_{1}\right)^{+}+\left(u_{1}+a_{1}\right)^{-}\right]
$$


or expressed in terms of $f_{1}$,

$$
V_{1}=\frac{1}{4}(\gamma+1)\left\{f_{1}^{+}\left(\alpha_{s}\right)+f_{1}^{-}\left(\alpha_{s}\right)\right\}+\frac{1}{2}(\gamma-3) f_{1}(\beta) .
$$

Therefore, along the shock

$$
d x_{s}=\left(1+\epsilon V_{1}\right) d t_{s}=d t_{s}+\epsilon \times \frac{1}{2} V_{1} d \beta .
$$

By integrating this equation from $A$ to $B$, and using the known values of $x_{s}(A)$ and $x_{s}(B)$, we get

$$
t_{s}(B)-t_{s}(A)=1+\epsilon\left[\hat{\omega}_{1}-\frac{\gamma+1}{2}\left(\frac{f_{1}^{+}+f_{1}^{-}}{2}\right)\right] .
$$

Note that, here, we cannot use the condition that $\left\langle f_{1}\right\rangle=0$, which was derived using the continuity of the solution. Repeating the process for the left-going shock $B A^{\prime}$, and combining both results, we get

$$
t_{s}\left(A^{\prime}\right)-t_{s}(A)=2+2 \epsilon\left\{\hat{\omega}_{1}-\frac{\gamma+1}{2}\left(\frac{f_{1}^{+}+f_{1}^{-}}{2}\right)\right\} .
$$

However, periodicity imposes that this difference be exactly 2, so the shock relation reduces to

$$
\frac{1}{2}\left(f_{1}^{+}+f_{1}^{-}\right)=2 \hat{\omega}_{1} /(\gamma+1) .
$$

This is, however, still not enough to determine the solution completely, as $\left\langle f_{1}\right\rangle$ is left unknown. We can get this information by integrating (3.17), in much the same way as with the continuous case, from just in front of the shock to just behind the shock one period ahead. Then

$$
\left\{\hat{\omega}_{1}-\frac{\gamma+1}{2} \frac{f_{1}^{+}+f_{1}^{-}}{2}\right\}\left[f_{1}^{-}-f_{1}^{+}\right]+b_{1}\left\langle f_{1}\right\rangle=0
$$

and using (4.15) we have

$$
\left\langle f_{1}\right\rangle=0, \quad \hat{\omega}_{1}=\omega_{1},
$$

for all cases except $b_{1}=0$, where (4.17) can be fixed by definition as before.

We may, then, simplify the shock condition with (4.17), and use both to construct discontinuous solutions by piecing together segments of continuous solutions of (4.1) with jumps satisfying (4.15). Detailed examples of wave forms constructed in this way are given by Chester (1964) for $b_{1}=0$, so we turn our attention to the more general case where $b_{1} \neq 0$.

We can expect that in those cases too it will be necessary to introduce shocks for some range of parameters, and, so, we must study first of all the question of existence of continuous solutions; this obviously depends on the behaviour of the singularities of (4.1). We study these singularities next.

They occur in the $f_{1}, \alpha$ plane when the coefficient of $f_{1}^{\prime}$ vanishes, or

$$
f_{1}=2 \omega_{1} /(\gamma+1)
$$

and the solution $f_{1}(\alpha)$ is only affected by them when it has to pass through that value. For large values of $\omega_{1}$, that can only happen for very large amplitude waves. However, the asymptotic solution (4.5) suggests that the amplitude really decreases as $\omega_{1}$ increases, so condition (4.18) is never realized for large $\omega_{1}$ and 


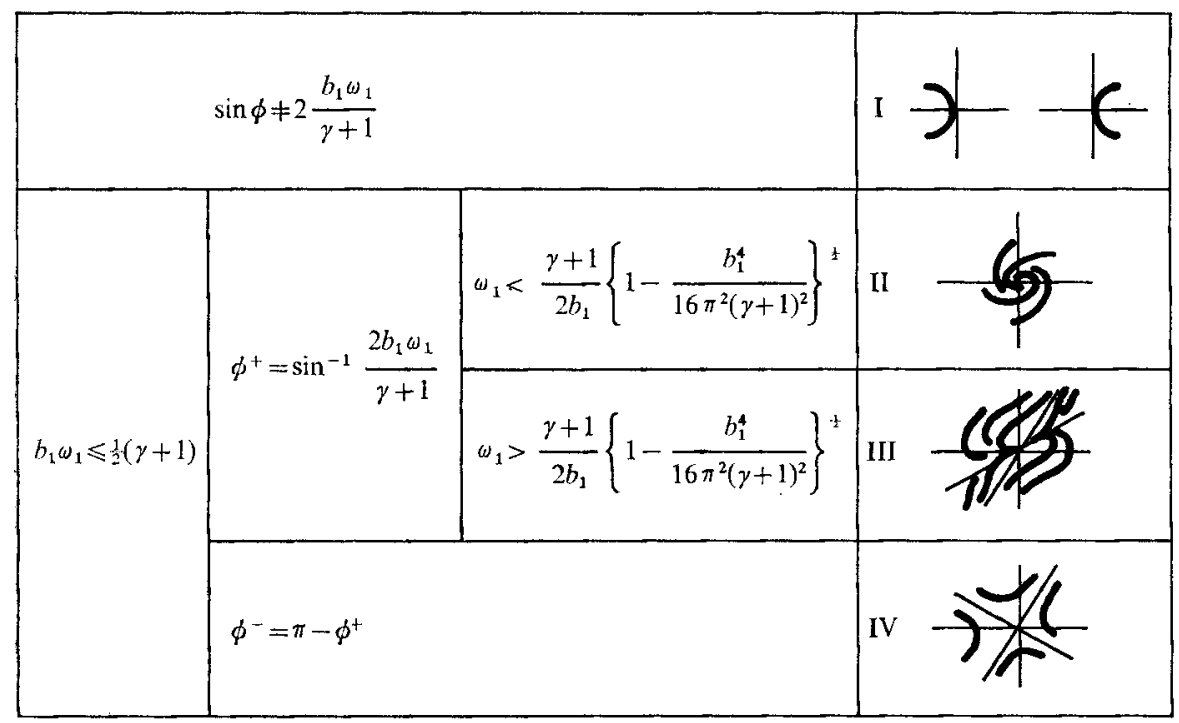

Figure 3

continuous solutions should be expected for that range. Once a continuous solution has been shown to exist, it is easy to convince oneself that it is unique.

As $\omega_{1}$ approaches zero, though, the wave amplitude increases and (4.18) decreases, so that at some sufficiently small frequency the solution will touch the singularity at some point. We need to consider, then, the behaviour of (4.1) near those points, and to do this we expand the equation for small displacements around (4.18). In particular, let

$$
f_{1}=2 \omega_{1} /(\gamma+1)+g, \quad \alpha=\phi+z,
$$

where $\phi$ is some general value of $\alpha$ in the neighbourhood of which we want to study the equation. Substituting in (4.1), we have

$$
(\gamma+1) g g^{\prime}-b_{1} g=\left(\frac{2}{\gamma+1} b_{1} \omega_{1}-\sin \pi \phi\right)-(\pi \cos \pi \phi) x+\ldots
$$

There are two possible cases, depending on the $\phi$ chosen. In most instances the constant on the right-hand side of $(4.20)$ is not zero, and the behaviour of the solution is then given by

$$
\left.\begin{array}{l}
(\gamma+1) g g^{\prime} \simeq\left(\frac{2}{\gamma+1} b_{1} \omega_{1}-\sin \pi \phi\right), \\
g \simeq \frac{2}{\gamma+1}\left[\left(b_{1} \omega_{1}-\frac{\gamma+1}{2} \sin \pi \phi\right) x\right]^{\frac{1}{2}} \cdot
\end{array}\right\}
$$

The exact shape of $g$ depends on the sign of the constant in round brackets, but it always includes a branch point at $x=0$, giving two-valued solutions which are inadmissible from a physical point of view (see figure 3 ).

If, however, $b_{1} \omega \leqslant \frac{1}{2}(\gamma+1)$, there are two points in every cycle at which the constant term in (4.19) vanishes. Name these points as

$$
\phi^{+}=\sin ^{-1}\left[2 b_{1} \omega_{1} /(\gamma+1)\right], \quad \phi^{-}=\pi-\phi^{+} .
$$




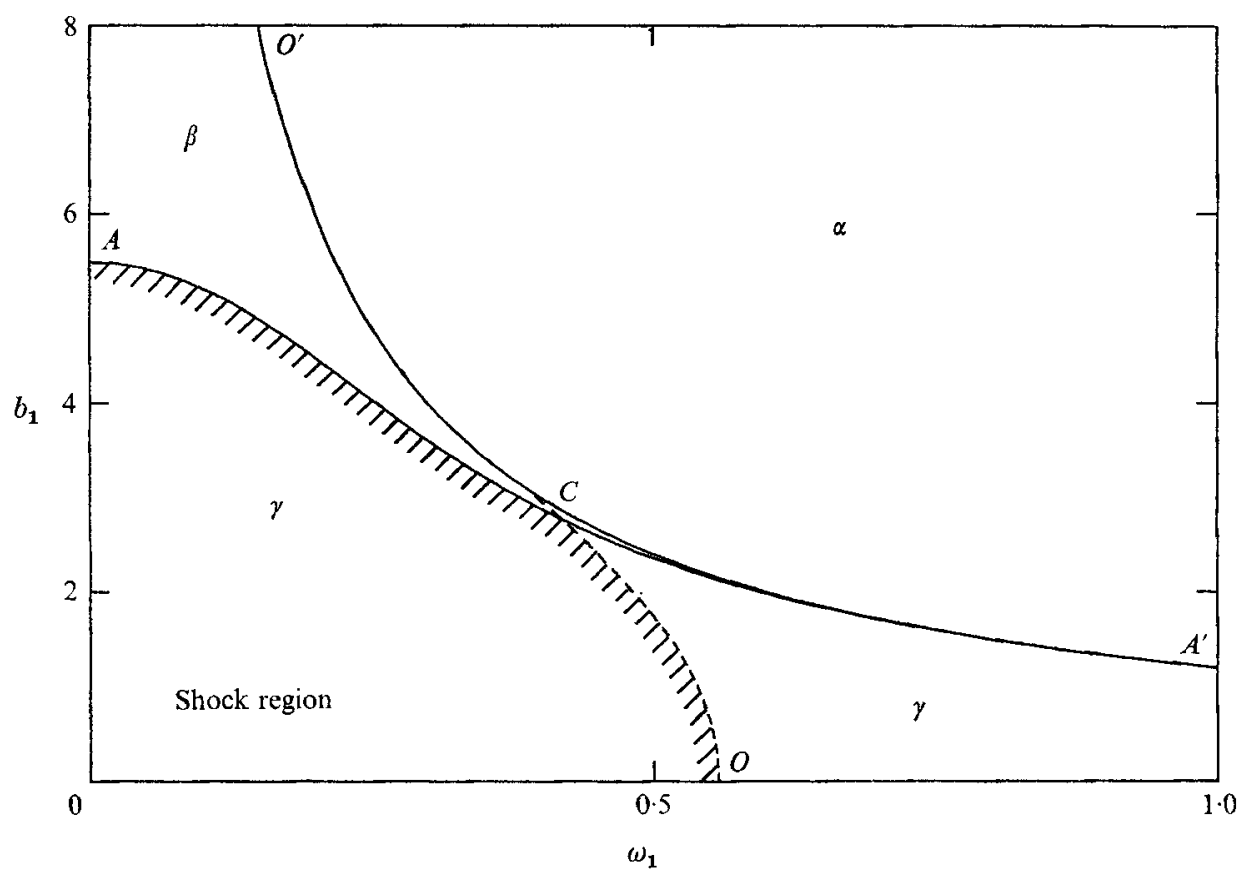

FIGURE 4. Division of the $b_{1}, \omega_{1}$ plane with regard to the nature of the singular points in the equation for the closed-end pipe.

The leading terms of (4.20) near those points are

$$
(\gamma+1) g g^{\prime}-b_{1} g+(\pi \cos \pi \phi) x=0 .
$$

This is now a bilinear equation, which can be solved exactly (Birkhoff \& Rota 1969, p. 13). The type of the solution depends on the behaviour of a particular quadratic algebraic equation involving the coefficients of (4.23). As the value of $\phi$ in (4.23) can be expressed through (4.22) in terms of $b_{1}$ and $\omega_{1}$, the nature of the singular points can be classified in terms of these parameters alone.

A summary of the most important results of this classification is given in figure 3. From this figure it is clear that, if a continuous solution is to cross the singular line (4.18) anywhere, it has to do it through one point of type III or IV. In fact, if it crosses the line at all, it has to cross it twice, once at $\phi^{+}$and again at $\phi^{-}$, and because of the shape of type III, it has to cross at $\phi^{+}$going up, and come back at $\phi^{-}$going down. If now, maintaining the same $b_{1}$, we start decreasing $\omega_{1}$, the point at $\phi^{+}$starts to 'roll-up' from type III to a spiral point of type II. At the moment that this happens, and $\phi^{+}$becomes of type II, the solution is no longer able to cross the singular line through that point, although it is still able to get back through $\phi^{-}$. At this moment an incipient shock develops at $\phi^{+}$and it grows bigger and bigger as the spiral rolls tighter with $\omega_{1}$ approaching zero.

Thus, the nature of the singular points divides the $b_{1}, \omega_{1}$ plane into regions, and in each region the possible types of solution are different. This classification is presented in figures 4 and 5. These, and all subsequent figures are drawn for air $(\gamma=1 \cdot 4)$, although a simple change in scale will adapt them for other values 

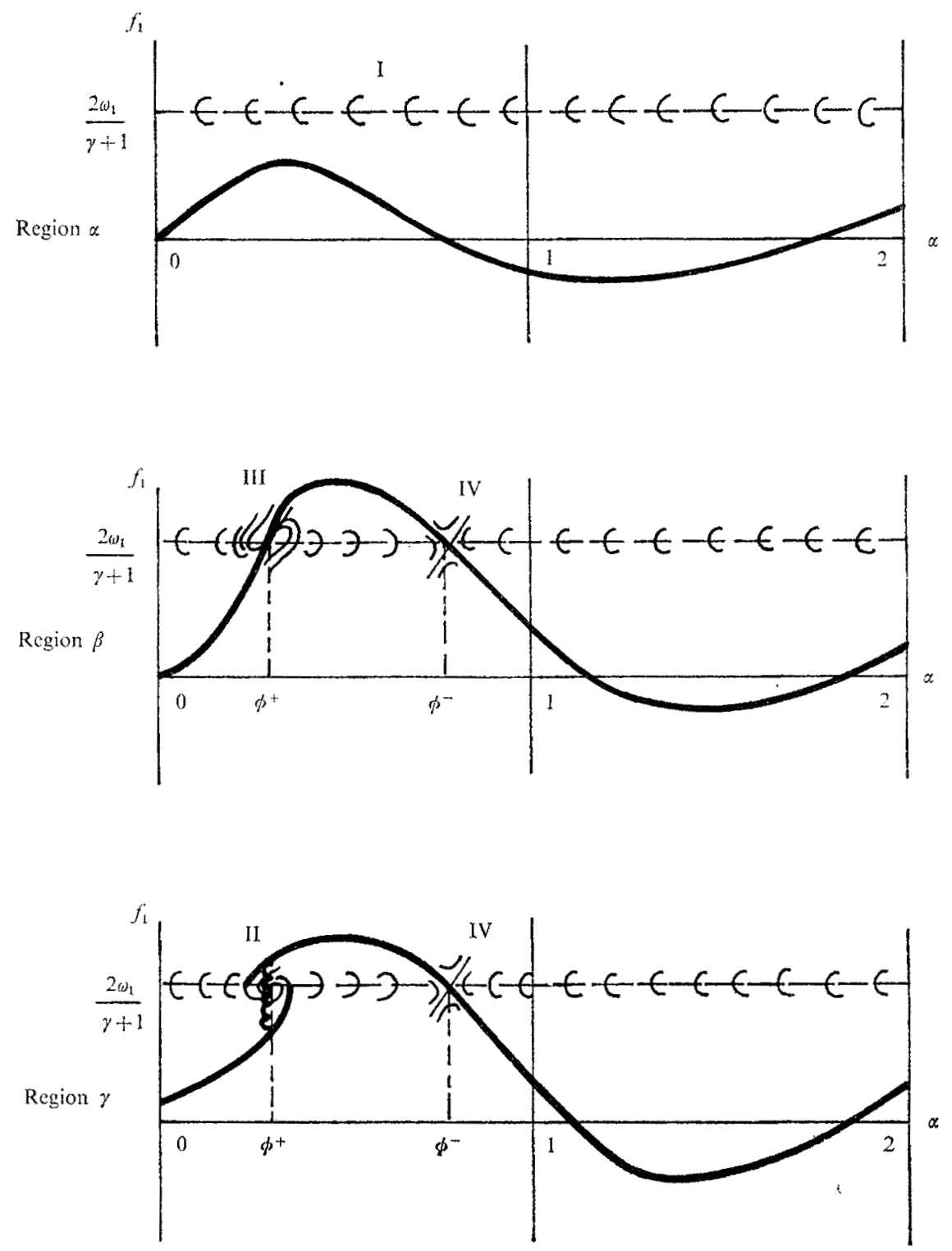

FIGURE 5. Character of the solution of (4.1) for the different types of singular point.

of $\gamma$. The wave forms given in figure 5 are those of the possible singular solutions in each region. In each case it is possible, in principle, to have also a continuous solution which never crosses the singular line, like that in region $\alpha$. The existence of this solution can be best decided by trying to integrate the equations numerically to find it.

The process used by us was a second-order 'shooting' scheme (Keller 1968, p. 54), starting from large $\omega_{1}$ for a fixed $b_{1}$ with the asymptotic solution (4.5) and working inwards keeping $b_{1}$ constant and decreasing $\omega_{1}$, until the solution touched the singular line. The points in the $b_{1}, \omega_{1}$ plane where the solution first 

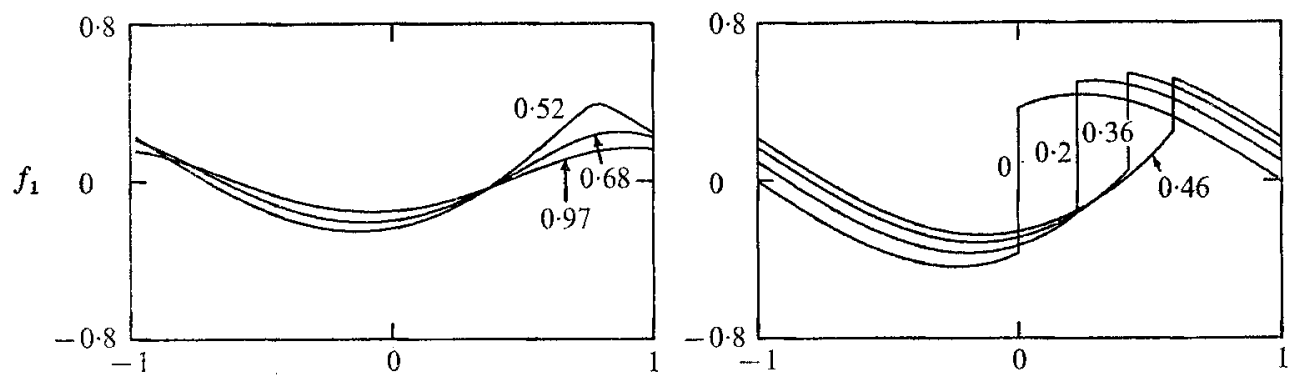

(a)
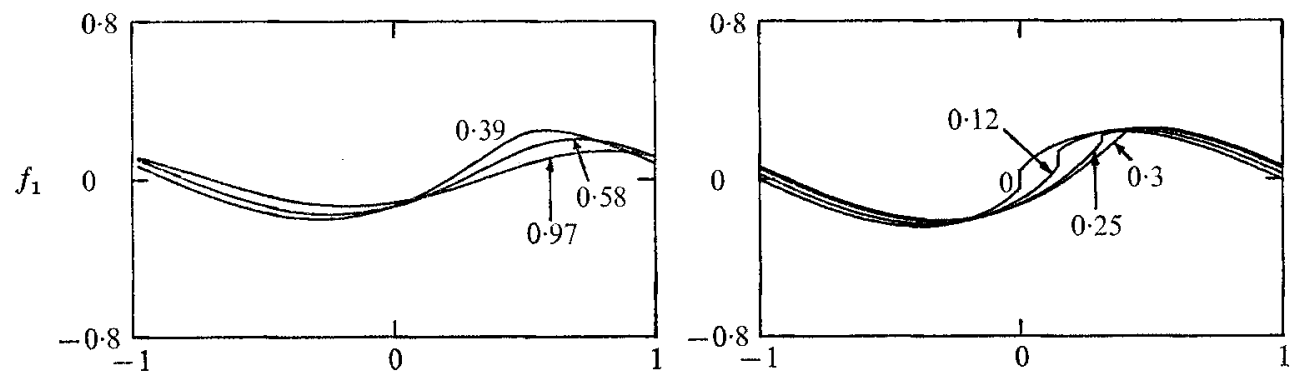

(b)
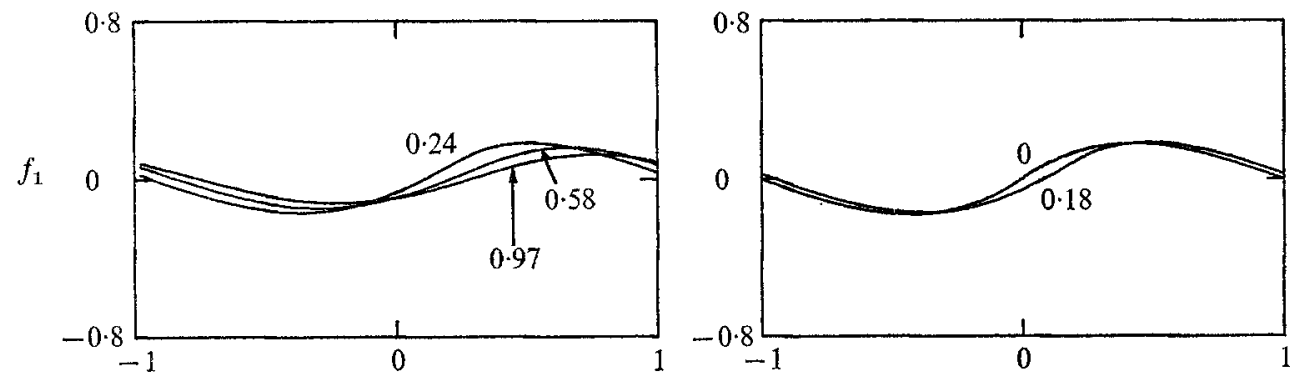

(c)

$\alpha$

$\alpha$

Figure 6. Typical wave forms computed for the closed-pipe case.

(a) $b_{1}=1 \cdot 55,(b) b_{1}=3 \cdot 87,(c) b_{1}=5 \cdot 42$.

touched this line are given by $O O^{\prime}$ in figure 4 . To the right of that line the solution is continuous and non-singular. To the left, the solution has to cross the singular line and the best way to find it numerically is to start integrating from $\phi^{+}$with the slope given by the analysis of the singular points and integrate forwards and backwards until the solution crosses the singular line again. A shock can then be fitted, if needed, using (4.15).

The calculations show that, in regions $\alpha$ and $\beta$, it is always possible to find a continuous solution for $f_{1}$, although the derivative may be discontinuous at $\phi^{-}$in some cases. The region of the $b_{1}, \omega_{1}$ plane where the solutions contains shocks is then only the part $O C A$ of region $\gamma$ lying to the left of $O O^{\prime}$.

All this discussion applies, of course, only to the case of $\omega_{1}>0$. For negative $\omega_{1}$ the results are completely symmetric, according to the transformation (4.6).

Finally, figure 6 gives some examples of wave forms computed for three 

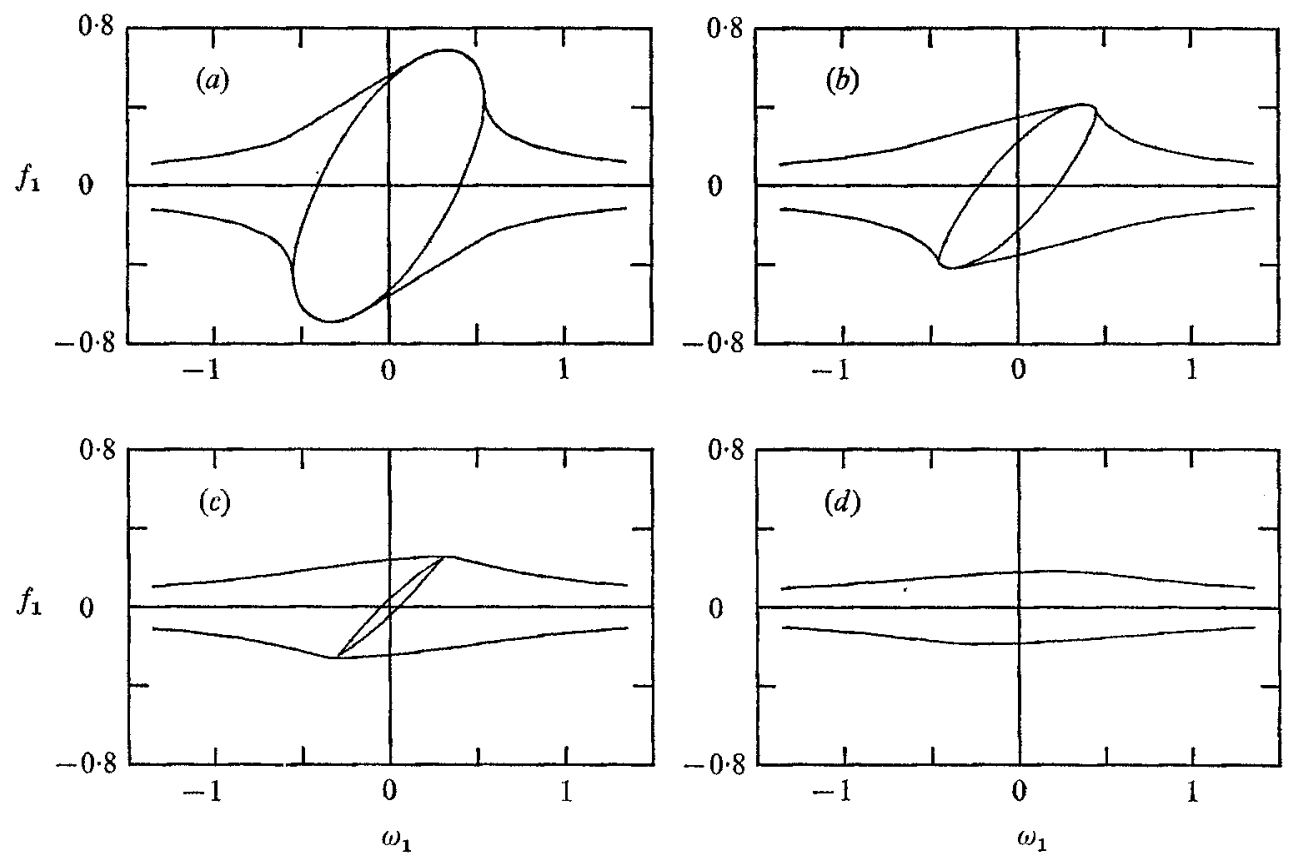

FIGURE 7. Wave amplitude for the closed pipe computed for several values of the radiation coefficient as a function of frequency. $(a) b_{1}=0 \cdot 77,(b) b_{1}=2 \cdot 32,(c) b_{1}=3 \cdot 87,(d) b_{1}=5.42$.

different values of $b_{1}$ and several $\omega_{1}$, using the method outlined above. Figure 7 is a plot of wave amplitudes as a function of $\omega_{1}$ for various values of $b_{1}$. The four quantities represented in each plot are respectively the maximum and minimum values of $f_{1}$, and the values at the top and foot of the shock.

It is to be noted that, although a shock is present in the solution for relatively large values of $b_{1}$, its strength becomes very small for much lower values of the radiation coefficients, to the point of being practically negligible for the larger $b_{1}$ 's.

\section{Results for the open-end case}

We now turn our attention to the pipe with the open end. The pertinent equation is $(3.24)$,

where

$$
\left.\begin{array}{c}
{\left[\hat{\omega}_{2}+\frac{1}{16}(\gamma+1)(3 \gamma+7) f_{1}^{2}\right] f_{1}^{\prime}+b_{2} f_{1}=\sin \pi \alpha,} \\
\hat{\omega}_{2}=\omega_{2}+\frac{1}{16}(3-\gamma)(13-3 \gamma)\left\langle f_{1}^{2}\right\rangle
\end{array}\right\}
$$

and is to be integrated in the interval $(0,1)$ subject to the condition

$$
f_{1}(1)=-f_{1}(0) \text {. }
$$

The results can be extended to the full period $(0,2)$ by using $(3.21)$,

$$
f_{1}(\alpha+1)=-f_{1}(\alpha) \text {. }
$$

The significance of the parameters $b_{2}$ and $\omega_{2}$ is similar to that of the corresponding ones in $\S 4$, and here too $b_{2}$ is essentially a non-negative number.

The whole problem is mathematically very similar to the closed-end case, and 
most of the analysis carries through directly to (5.1). The singularities in this case are located at

$$
f_{1}= \pm 4\left\{-\widehat{\omega}_{2} /(\gamma+1)(3 \gamma+7)\right\}^{\frac{1}{2}}
$$

so they form two singular lines, instead of one. From (5.4), too, these lines are only real when $\hat{\omega}_{2} \leqslant 0$. For $\omega_{2}$ positive, we can expect no trouble with singularities and the solutions are continuous and well behaved; this is of course also true for $\hat{\omega}_{2}$ sufficiently negative.

For intermediate values of the frequency, however, the solution crosses the singular lines and exhibits the same kind of phenomena as the closed-end solution. The analysis of the singular points runs exactly parallel to the one there and, in fact, the approximate equation near (5.4) is also bilinear in this case, the only difference being that the functions of the points $\phi^{+}$and $\phi^{-}$are interchanged. The fact that there are two singular lines instead of one does not affect the results much, because it turns out that each line interacts with only one half-period of the wave form, as could be suspected from (5.3). In particular, the interval $(0,1)$ is only involved. with the plus sign in (5.4).

There are, however, several important differences between this and the closedend case. The first one is the existence of a shift between the effective frequency $\hat{\omega}_{2}$ and the physical quantity $\omega_{2}$. This shift vanished in the closed pipe, as we were able to show that $\left\langle f_{1}\right\rangle$ was always zero. In (5.1), however, the shift depends on $\left\langle f_{1}^{2}\right\rangle$, which is a strictly positive number.

The significance of this shift was discussed in $\$ 3$, and its effect in the system is to tilt the resonance peak toward lower frequencies, this effect being bigger as $b_{2}$ becomes smaller and the wave amplitudes grow larger.

Numerically, of course, all the work is done first using $\hat{\omega}_{2}$ as a parameter, and after the solution has been found, $\omega_{2}$ is computed using (5.1). The results presented in this section are for $\gamma=1.4$, but, owing to (5.1), the change to other gases no longer corresponds to a simple change in scale.

The division of the $b_{2}, \hat{\omega}_{2}$ plane according to the type of singularities is plotted in figure 8, where the names of the regions correspond roughly to the descriptions given in $\$ 4$. The solution passes through the singular lines in the region between the line $O O^{\prime}$ and the $b_{2}$ axis. The only region qualitatively different from the closed case is the one to the right of the ordinate axis, where no singularities exist and the solution is always continuous. The shock region is given by the area of region $\gamma$ to the right of $O O^{\prime}$ and, in figure 9, it is plotted in 'physical' $b_{2}, \omega_{2}$ co-ordinates.

A very important difference from $\S 4$ occurs however in the behaviour of the solution within the shock region. The main reason for it lies in (5.3), for this equation assures that anything that happens in one semiperiod will happen with the opposite sign half a period later. In particular, any compression shock in the wave must be followed by an expansion shock of the same strength, which is physically quite unlikely.

Even more important is the fact that (5.1) really derives from the equations of motion used up to $O\left(\epsilon^{3}\right)$, and assumed isentropic. Now, the entropy production of a shock of amplitude of $O(\epsilon)$ first appears in the equations at $O\left(\epsilon^{3}\right)$, so that the assumption of isentropic flow is inconsistent with the existence of shocks. This 


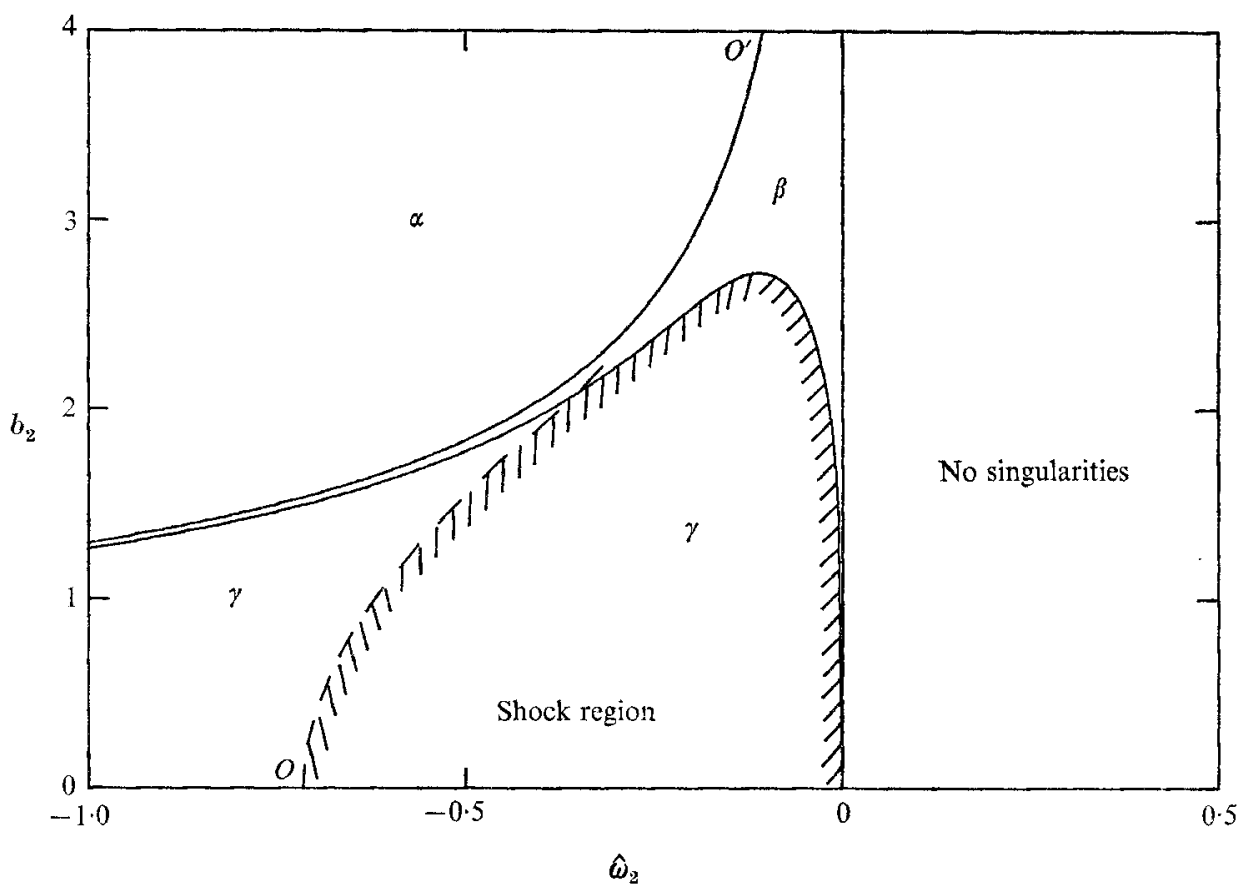

Frgure 8 . Division of the $b_{2}, \hat{\omega}_{2}$ plane with regard to the nature of the singular points in (5.1).

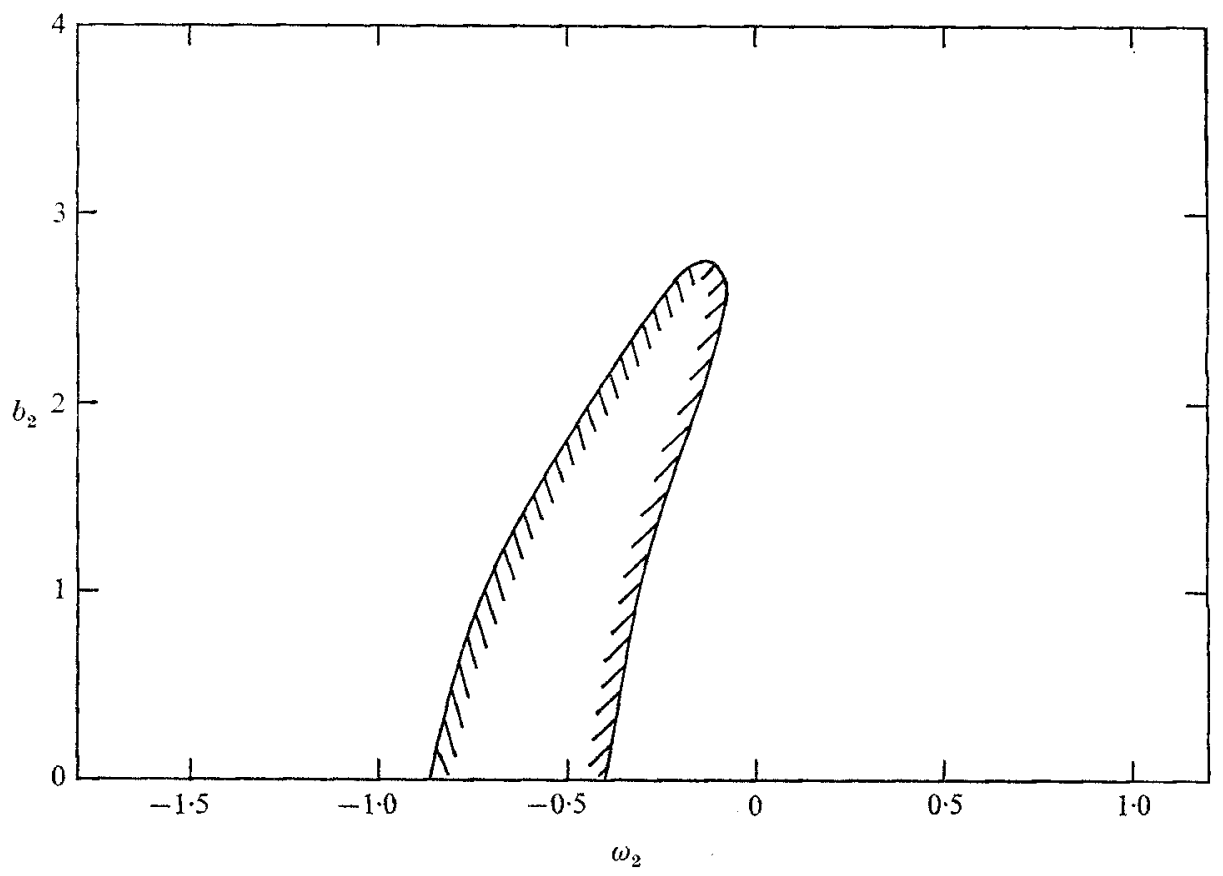

FigURE 9. Extent of the region containing shocks in the solution of (5.1), in the $b_{2}, \omega_{2}$ plane. 
is reflected mathematically in the impossibility of finding any condition for a discontinuity in the solution to represent a shock, equivalent to (4.15) for the closed case. In fact, as we try to repeat the process in $§ 4$, to get this kind of condition, we run into the difficulty that any effect produced by the shock is cancelled by the opposite expansion shock somewhere during the period.

The influence of the entropy production on the solution has another effect on the attempt to formulate a physical model including this influence. The flow in the pipe is supposed to be periodic, so that the entropy produced by one passage of the shock at any one point has to be removed somehow before the next passage. The way this entropy is removed is, of course, by cooling the fluid through the walls of the tube, and the modelling of this cooling depends on the exact experimental set up, and introduces new parameters into the problem.

To avoid these complications, and in view of the fact that the reflexion condition is probably not very good for shocks at an open end, we decided to abandon any attempt to compute discontinuous wave forms in this case. The boundary of shock formation, plotted on figure 9 , should remain valid, however, as it is essentially a negative result establishing the impossibility of continuous solutions.

Some representative examples of wave forms computed for three different values of $b_{2}$ and several frequencies are given in figure 10 . For the first two values of $b_{2}$ the solution cuts across the shock region so that only results for frequencies on either side of the boundary are shown; in those cases, the tendency for shocks to form can already be seen quite clearly. The third value of $b_{2}$ is above the shock boundary and so the wave form can be computed for all frequencies and is always continuous. In figure 11, the half-amplitude of the wave is plotted versus frequency with $b_{2}$ as a parameter. The gaps in the curves correspond to regions with shocks.

The most important result in connexion with the open-end case is, however, connected with orders of magnitude. Going back to the definition of $\delta$ as measuring the amplitude of the piston motion and $\epsilon$ as measuring the strength of the gas motion, we see that for the closed end $\epsilon=\delta^{\frac{1}{2}}$, while for the open end $\epsilon=\delta^{\frac{1}{3}}$, thus the oscillation is in fact stronger when the end is open.

This result, which may seem somewhat surprising at first sight, is however easily explained. The effect of a closed end on an incoming wave is to reflect it with the same sign in pressure. Thus, a compression wave is reflected as a compression wave, and never changes sign. Consider now a small pressure step produced, say, at the piston in a closed pipe. If the step was initially a compression, it remains a compression for ever, and the steepening of the wave, that is the interaction of the wave with itself, acts continually and eventually becomes important. This nonlinear effect appears in the equations at $O\left(\epsilon^{2}\right)$ and helps to 'kill' the linear resonance.

For an open end the sign of the wave in the pipe changes every time it is reflected at the open section, and so any particular signal is a compression half of the time and an expansion the other half. So, the steepening by interaction of the wave with itself never accumulates, and it is only after third-order interactions come into play that the linear resonance can be limited. 

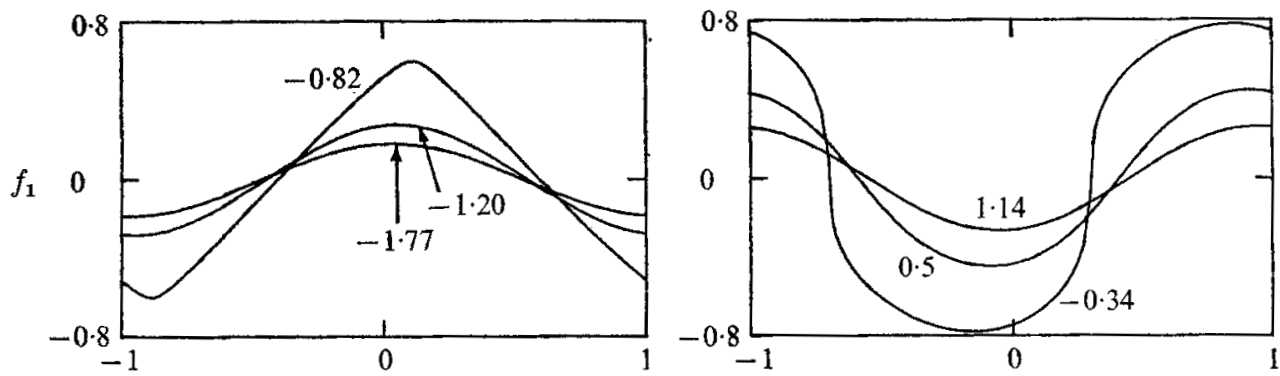

(a)
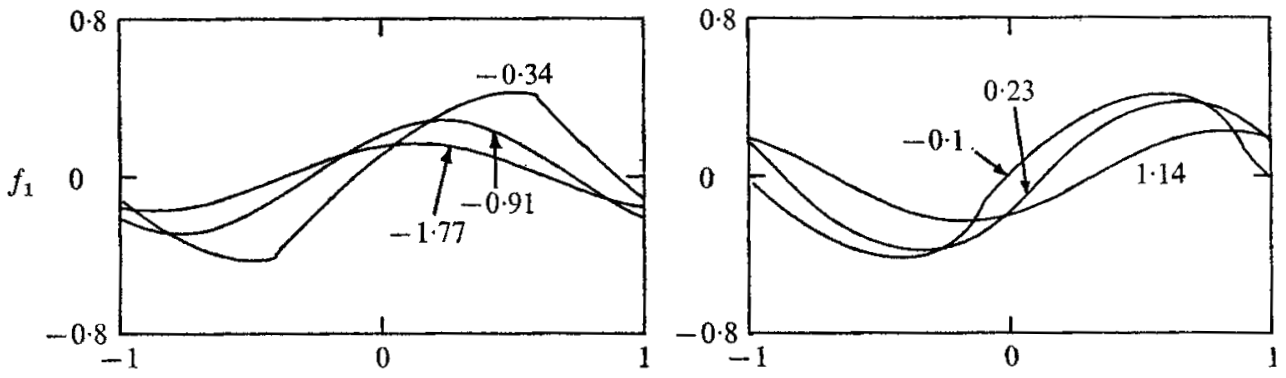

(b)
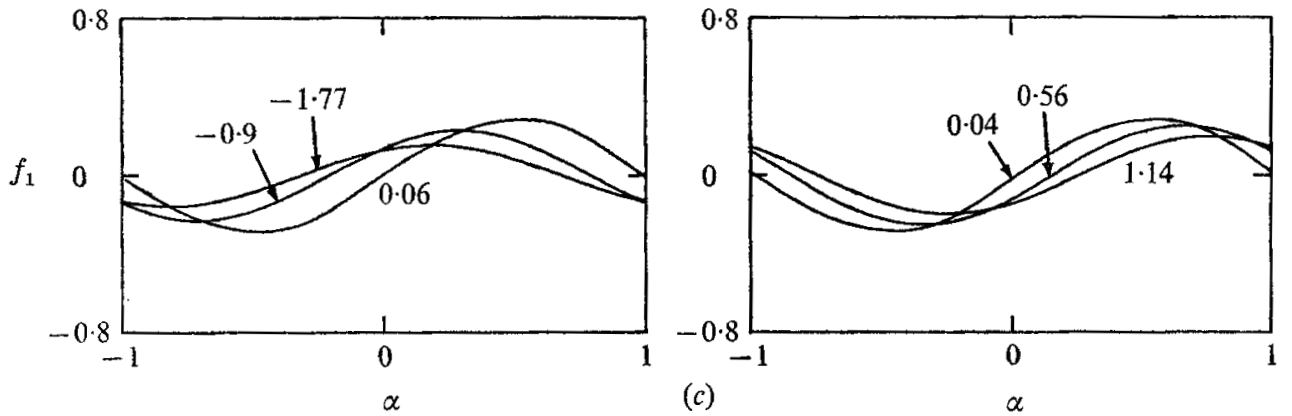

Figure 10. Typical wave forms computed for the open-pipe case. (a) $b_{2}=0.59$. (b) $b_{2}=2 \cdot 34$. (c) $b_{2}=3 \cdot 51$.

It is important to realize on the other hand that, from the energy point of view, an ideal open end is as closed as a rigid wall. In fact the energy flow out of the end section is given by

$$
\dot{E}=\oint p d V
$$

where $V$ is the volume of the gas originally in the tube. For the closed pipe $d V=0$ for all time and the energy flows vanishes. But for the open pipe the pressure is constant at the exit so

$$
\dot{E}=p \oint d V=p \Delta V=0
$$

by periodicity, and the energy flow vanishes too. 


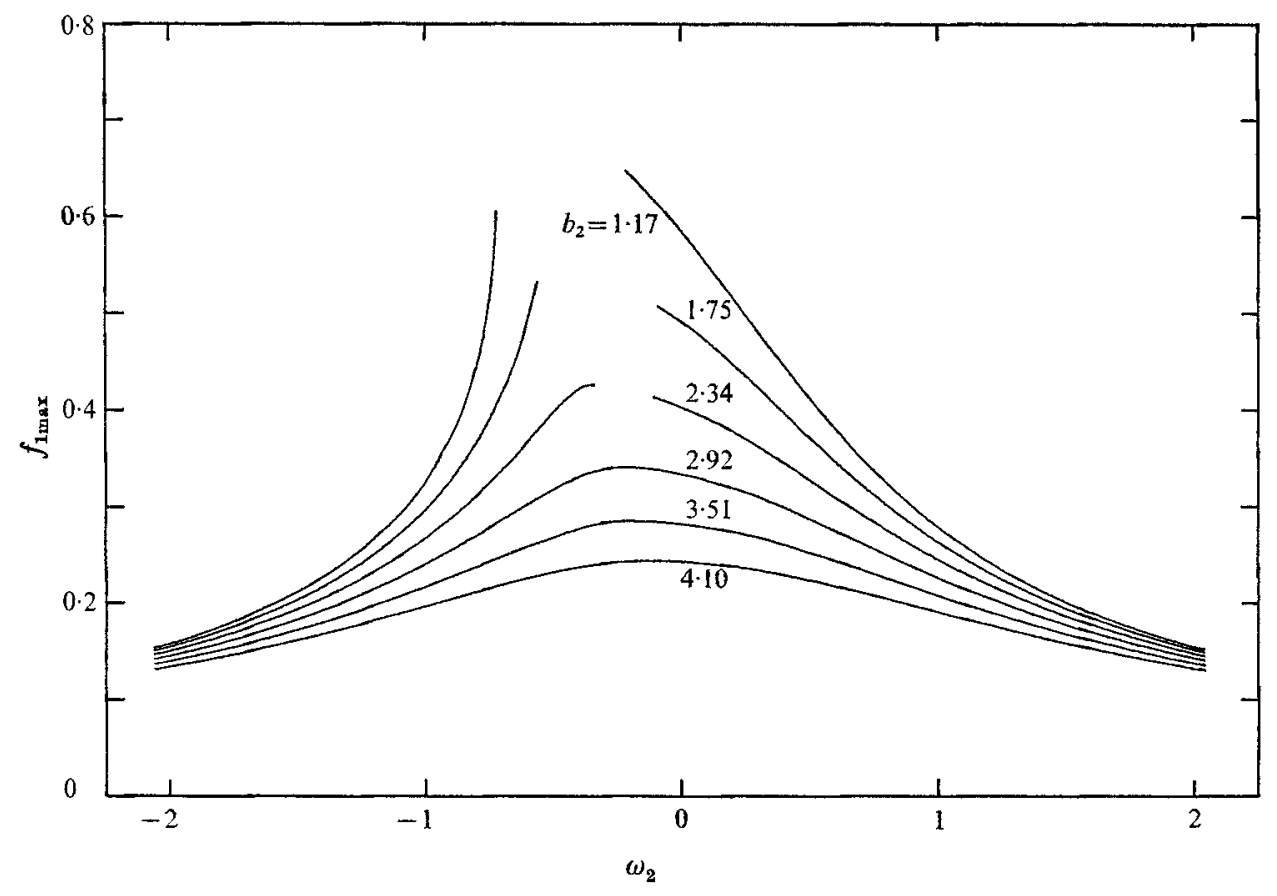

Fraure 11. Wave amplitude for the open pipe computed for several values of the radiation coefficient as a function of the frequency.

Therefore, the only remaining factor to decide the strength of the wave is the order of the nonlinearity, and a weaker effect, like the one in the open end, will not limit the resonance peak until the higher amplitudes necessary to make the nonlinearity important are attained.

In the real world, of course, open ends do radiate a lot of power, which means that the perfect open end, $b_{2}=0$, is probably a limiting case with no physical reality.

\section{Conclusions}

The breakdown of linear acoustic theory at resonance in both closed and open pipes can be remedied by appeal to higher order nonlinear effects. A consistent perturbation analysis of the nonlinear equations is presented for the case of oscillations produced by the sinusoidal motion of a piston in one end of the pipe. At the other end of the pipe, it is supposed that the wave profile reaching that end is reflected with a factor $b$. The reflexion coefficient ranges from $b=1$ for the completely closed end to $b=-1$ for the 'ideal' open end used in acoustics. Resonance occurs for $b$ in the neighbourhood of $b=1$ and $b=-1$. Particularly for the nearly open end, this boundary condition is obviously a severe simplification of a complicated situation. In fact, the reflexion characteristics may depend on the frequency, the shape of the particular wave profile and so on. The attitude here is not, however, to insist that the reflexion is independent of these influences, but rather to learn about the 'equivalent $b$ ' by comparison of the results with 
experiment. As will be reported in part 2, it is possible to correlate theory and experiment in this way, and indeed the theoretical results are invaluable in developing a correct rational interpretation of the experiments for a variety of input conditions and end conditions. This information on the effective reflexion coefficient, its dependence on frequency, etc. should be valuable in other situations.

For the open and nearly open pipes the amplitude of the oscillations in the pipe are of $O\left(\delta^{\frac{1}{3}}\right)$, where $\delta$ is the piston amplitude. This is in marked contrast to the result $O\left(\delta^{\frac{1}{2}}\right)$ obtained by earlier investigators for the closed-end case. In the closed or nearly closed cases, $b \approx 1$, the result stems from a balance between nonlinear steepening and forcing by the piston. For the open-end cases $b \approx \mathbf{- 1}$, however, the second-order distortion effect alternates in sign for the successive runs up and down the tube, and the forcing can only be balanced by terms of third order in the gas amplitude. The resulting amplitude, proportional to $\delta^{\frac{1}{3}}$, is then higher than in the closed-end case. This is confirmed by experiment for appropriate ranges of the parameters. The detailed comparison of this and other predictions with the experimental observations will be given in part 2 .

This work was motivated by the experiments on nonlinear resonance being conducted by Professor B. Sturtevant of this Institute. The experimental results will be reported in subsequent parts of this series.

The work was supported in part by a contract with the office of Naval Research (N00014-67-A-0094-0014) and a grant from the National Aeronautics and Space Administration (NGR 05-002-220) and the author was supported partially by a fellowship from the European Space Research Organization.

\section{REFERENCES}

Betchov, R. 1958 Phys. Fluids, 1, 205.

BtrжноғF, G. \& RotA, G.-C. 1969 Ordinary Differential Equations. Waltham, Mass.: Blaisdell.

Chester, W, $1964 J$. Fluid Mech. 18, 44.

Chu, B.-T. \& YING, S. J. 1963 Phys. Fluids, 6, 1625.

Coumins, W. D. 1971 Quart. J. Mech. Appl. Math. 24, 129.

Courant, R. \& Friedrichs, K. O. 1948 Supersonic Flow and Shock Waves. Interscience.

InGard, U. \& Ising, H. 1967 J. Acoust. Soc. Am. 42, 6.

Kellen, H. B. 1968 Numerical Methods for Two-Point Boundary Value Problems. Waltham, Mass.: Blaisdell.

LetTat, E. 1939 Dtsch. Kraftfahrtforsch. 39, 1.

Lin, C. C. 1954 J. Math. \& Phys. 33, 117.

Morse, P. M. \& Ingard, K. U. 1968 Theoretical Acoustics. McGraw-Hill.

Morted, M. P. 1971 Int. J. Engng Sci. 9, 175.

Rayleigh, LoRd 1945 The Theory of Sound. Dover.

Saenger, R. A. \& Hudson, G. E. 1960 J. Acoust. Soc. Am. 32, 961.

Seymour, B. R. \& Mortel, M. P. 1973 J. Fluid Mech. 58, 353.

Temkin, S. 1968 Phys. Fluids, 11, 960.

WiJngaARDEN, L. VAN 1968 J. Engng Math. 2, 225. 